\title{
Percepción de conductas abusivas en estudiantes de medicina
}

\author{
Ana Margarita Maida $\mathbf{S}^{1}$, Viviana Herskovic $\mathbf{M}^{2}$, Ana Pereira \\ $\mathrm{S}^{3}$, Lorena Salinas-Fernández ${ }^{a}$, Claudia Esquivel $\mathrm{C}^{\mathrm{a}}$. \\ Perception of abuse among medical \\ students of the University of Chile
}

Background: Even though studying Medicine and perceiving abuse seem to be two opposite situations, recent investigations in Chile and abroad find that this is a frequent and pervasive combination. These studies also report the negative effects in the lives of students as well as the impact on the profession as a whole. Aim: To ascertain the perception of abusive situations in medical students during training at the University of Chile. Material and Methods: Descriptive and cross sectional study in which a questionnaire was applied to all the students enrolled in $2^{\text {nd }}, 3^{\text {rd }}, 5^{\text {th }}$ and $7^{\text {th }}$ year during 2001 and 2002. Using short vignettes, they were asked if they had experienced verbal, psychological, physical and sexual abuse, at least once, during their training, by whom and the eventual effects derived from it. Results: We obtained 757 questionnaires. Of the surveyed students, 91\% reported having perceived at least one abusive episode during training. Teachers and fellow students were identified as the main offenders. Among the effects of such behavior, $32 \%$ mentioned that they considered dropping out of the career as a consequence of this experience. Discussion: The perception of abuse in medical students is common and has adverse effects. Efforts should be made to draw attention to this problem to prevent it (Rev Méd Chile 2006; 134: 1516-23).

(Key words: Education, medical; Sexual abuse; Students, medical)

Recibido el 2 de noviembre, 2005. Aceptado el 30 de mayo, 2006.

El trabajo realizado fue aprobado por ASOFAMECH, en 2002, como parte de estudio multicéntrico.

${ }^{1}$ Departamento de Pediatría y Cirugía Infantil Oriente, Facultad de Medicina, Universidad de Chile, Hospital Luis Calvo Mackenna.

${ }^{2}$ Clínica Psiquiátrica Universitaria, Universidad de Chile, Santiago de Chile.

${ }^{3}$ Escuela de Salud Pública, Universidad de Chile.

anterna, Facultad de Medicina, Universidad de Chile.

A busar es tratar a otro en forma ofensiva, causar lesiones físicas o psicológicas, o forzar a otro a realizar acciones que no desea 0 en las

Correspondencia a: Dra. Ana Margarita Maida S. Antonio Varas 360, Providencia, Santiago, Chile. Fonos: 2356915, 3401751, 2731415, 2739897. Fax: 2731415.

E mail: mmaida@med.uchile.cl que no conciente. Una conducta, para considerarse abusiva, debe darse en un contexto de desequilibrio de poder. La percepción de abuso es un hecho subjetivo ${ }^{1}$.

Aunque pudiera parecer que la combinación de estudiar medicina y sufrir abuso es algo contradictorio, el reporte anecdótico e investigaciones recientes en el tema, tanto en Chile como 
en el extranjero, dan cuenta que es un fenómeno frecuente ${ }^{1-4}$ (e Ibáñez P., III Congreso Internacional de Educación en Ciencias de la Salud, Santiago, 2005; Pantoja M.A. en el mismo congreso).

Hasta no muchos años atrás, sólo había referencias informales sobre situaciones abusivas en la carrera de medicina y los médicos solían recordar episodios aislados en reuniones de ex-alumnos, pero no existían datos al respecto. A partir de un comentario publicado en Journal of the American Medical Association (JAMA) por Henry Silver en $1982^{5}$, surgió el interés de estudiar esta situación. Él opinaba que dos estudiantes aprenden no lo que los profesores dicen, sino lo que hacen»y que la presencia de situaciones abusivas era destructiva para la educación médica»

Sheehan reportó que los estudiantes percibían que las conductas de abuso eran muy frecuentes y generalizadas durante la carrera de medicina, mencionando a los docentes y a sus pares como las principales fuentes de agresión ${ }^{7}$. En el estudio de 1990, Silver encontró que $80,6 \%$ de los estudiantes que reportaban algún tipo de abuso, consideraban que estos episodios habían sido «muy perturbadores» y que $16,2 \%$ referían que «siempre se iban a sentir afectados por ello». En 1998, Schuchert encontró que las situaciones abusivas afectaban al estudiante en la seguridad en sí mismo y en sus habilidades clínicas $^{8}$. Richman, Carr, Oancia y White coinciden en que las mujeres se sentían más afectadas por las situaciones de abuso ${ }^{9-12}$. Setenta y cinco por ciento de los alumnos encuestados por Sheehan informó haberse convertido en personas cínicas con respecto a la vida académica y la profesión médica7. Es más, Kassebaum reportó que los estudiantes opinaban que sasí se daba la educación en medicina» y manifestaban su preocupación por la insensibilidad que podía transmitirse de generación en generación ${ }^{13}$.

Sufrir situaciones de abuso, en cualquier etapa de la vida, es una experiencia traumática ${ }^{14-16}$. Quienes lo han experimentado y, más aún si es causado por personas cercanas, conocen la situación de dolor y aislamiento que acompañan al abuso. No es menor el sufrimiento si la situación abusiva se produce en una institución educacional. Educar implica formar, conducir, guiar a otro por lo que la enseñanza debiera ir acompañada de la entrega de conocimientos y valores que permiten el desarrollo del individuo. Sufrir abuso por parte de los profesores deforma el proceso educacional en un sentido profundo. Si se espera que los alumnos aprendan a respetar a otros, el mensaje que se entrega tiene que ser coherente, $\mathrm{e}$ ir acompañado de acciones que lo demuestren. $\mathrm{Si}$ el mensaje explícito es «ienes que ser respetuoso» y el implícito es «e desprecio», se instaura una experiencia psicológica que puede ser muy difícil de elaborar.

En recientes conferencias de Educación en Ciencias de la Salud (Conferencias Ottawa, Congresos AMEE, Congresos de Educación en Ciencias de la Salud celebrados en Chile), se ha reiterado la importancia de volver a enseñar medicina desde una perspectiva humanista, en que el médico no sea tan solo un tecnócrata, sino quien acompaña al enfermo en momentos de aflicción, quien lo comprende y se compadece ante el sufrimiento. Junto al bagaje de conocimientos, se espera que los futuros médicos aprendan a ser más tolerantes, empáticos y compasivos ${ }^{17}$.

Cada vez hay más información con respecto a las consecuencias que sufren las víctimas de abuso, que va desde alteraciones menores a cuadros psiquiátricos graves ${ }^{14-16,18}$. Las víctimas han empezado a relatar sus experiencias y a dejar atrás el silencio. El reconocimiento de las situaciones de abuso no sólo produce una sensación de desagravio en las víctimas, sino que se asocia también a una disminución de las conductas abusivas por parte de los agresores ${ }^{16}$.

La ocurrencia de conductas abusivas en la escuela de medicina podría ser particularmente grave. Si un estudiante percibe intolerancia, agresión o abuso, ¿qué efectos tiene esta experiencia psicológica? Sheehan, Kassebaum e Ibáñez estiman que percibir situaciones abusivas durante el entrenamiento médico puede contribuir en perpetuar el ciclo del abuso 2,13 (e Ibáñez P.).

La motivación de este estudio nace a partir del reporte de experiencias de esta índole en la Conferencia Ottawa 2000, y de incidentes abusivos aislados reportados a las autoridades de la Escuela de Medicina de la Universidad de Chile. Para documentar esta situación, se formó un equipo de trabajo, que realizó un estudio piloto de percepción de conductas abusivas en estudiantes de medicina ${ }^{2}$. Dados los resultados de este 
trabajo, ASOFAMECH propuso ampliar esta línea de investigación.

El presente trabajo tiene como objetivo estudiar la percepción de conductas abusivas a lo largo de la carrera de medicina, el tipo de abuso experimentado, quiénes lo efectuaron y qué efectos causaba en una muestra amplia de estudiantes.

\section{Material y MÉTOdO}

En la Escuela de Medicina de la Universidad de Chile se realizó un estudio descriptivo, transversal al universo de los estudiantes de $3^{\circ}, 5^{\circ}$ y $7^{0}$ años en 2001 y de $2^{\circ}, 3^{\circ}$ y $5^{\circ}$ en 2002 . El instrumento utilizado fue la Encuesta de Percepción de Conductas Abusivas (EPCA), perfeccionada a partir del estudio piloto por el equipo de trabajo en que participaban dos docentes y 5 estudiantes. EPCA es una encuesta autoaplicada, voluntaria y anóni$\mathrm{ma}^{2}$. La aplicación de EPCA fue aprobada por el Comité de Ética de la Facultad de Medicina de la Universidad de Chile. Para asegurar la confidencialidad de las respuestas, la encuesta fue devuelta en sobre cerrado, sin identificación del estudiante, con firma previa de consentimiento informado en una hoja aparte.

La variable de interés, 〈abuso», se definió de la siguiente forma en EPCA: «Abusar es tratar a otro en forma ofensiva, injuriosa o dañina; causar lesiones físicas o psicológicas; atacar con palabras; difamar; hablar en forma insultante, dura o injusta a una persona o acerca de ella». Una conducta abusiva sse da en un contexto de desequilibrio de poder, permanente o momentáneo (sea éste físico, psicológico, económico o político). El desequilibrio de poder implica la existencia de roles complementarios (como padre/hijo, hombre/mujer, maestro/alumno, patrón/empleado, joven/viejo) y no es necesariamente objetivable por un observador externo. El abuso provoca algún tipo de daño o menoscabo en la integridad de la persona que lo sufre $\aleph^{15}$. Hay situaciones de desequilibrio de poder que puede darse entre pares, motivadas por diferencia de edad, género, de número, de personalidad, contextura física y nivel socioeconómico.

Se solicitó información con respecto a la percepción de situaciones consideradas abusivas por los investigadores, en el plano verbal, psicoló- gico, físico y sexual mediante viñetas breves. Los estudiantes debían marcar si lo habían experimentado una vez, más de una vez o nunca. Si lo habían experimentado, debían anotar por parte de quiénes, cómo los había afectado y si sentían que el efecto iba a ser permanente. Además, se dio espacio para el reporte espontáneo de percepción de situaciones abusivas no mencionadas en la encuesta o si existían efectos distintos a los anotados por los autores al confeccionar la encuesta.

Para el análisis estadístico se utilizó el programa STATA 8.0, determinándose las frecuencias de conductas abusivas, agresores y efectos, buscándose asociaciones con género y curso, mediante la prueba de $\chi^{2}$, con un nivel de confianza de $95 \%$.

\section{Resultados}

Se contabilizaron las respuestas de 757 encuestas, correspondientes a $498(77,5 \%)$ de 2001 y 259 $(45,4 \%)$ de 2002 de los alumnos matriculados. De ellos, $48,5 \%$ eran hombres y $51,5 \%$ mujeres.

Durante la carrera de medicina, $9,1 \%$ de los estudiantes no percibieron situaciones abusivas, $4,8 \%$ refieren haber percibido sólo un incidente abusivo y $86,1 \%$ reportan percibir dos o más incidentes abusivos.

De los distintos tipos de abuso percibidos, el más frecuente fue el trato humillante, tanto verbal como psicológico (Tabla 1). Las mujeres reportan percibir significativamente más agresiones sexuales que los hombres; éstos, a su vez, perciben haber sido objeto con mayor frecuencia de agresiones físicas. No hubo diferencias significativas en los resultados de los años 2001 y 2002.

Cursos. La percepción de conductas abusivas muestra una tendencia significativa al aumento en cursos sucesivos, tanto en total, como al analizar por separado el abuso verbal, psicológico y sexual. La frecuencia de percepción de abuso físico no presenta esta tendencia (Tabla 2).

Agresores. Los docentes y los estudiantes fueron señalados como fuentes frecuentes de conductas abusivas. Los profesores fueron mencionados con una frecuencia significativamente mayor en rela- 
Tabla 1. Percepción de situaciones abusivas

\begin{tabular}{|lrrrr}
\hline & \multicolumn{2}{c}{ Una vez $^{1}$} & \multicolumn{2}{c|}{ Más de una vez } \\
& $\mathrm{n}$ & $\%$ & $\mathrm{n}$ & $\%$ \\
\hline Abuso verbal & & & & \\
$\quad$ Le han gritado & 115 & 15,1 & 190 & 25,1 \\
Llamado en forma abusiva, discriminatoria, insultante & 67 & 8,8 & 108 & 14,2 \\
$\quad$ Dicho garabatos en forma ofensiva & 32 & 4,2 & 45 & 5,9 \\
$\quad$ Sentido humillado por la forma en que han hablado & 193 & 25,5 & 370 & 48,9 \\
Abuso psicológico & & & & \\
Amenazado con perjudicar académicamente & 142 & 18,7 & 74 & 9,8 \\
Han hablado mal de usted para perjudicarlo & 88 & 11,6 & 100 & 13,2 \\
Le han asignado tareas injustas & 50 & 6,6 & 34 & 4,5 \\
Otros se han acreditado con su trabajo injustamente & 113 & 14,9 & 132 & 17,4 \\
Han ignorado o descalificado su trabajo & 138 & 18,2 & 193 & 25,5 \\
Le han tratado en forma humillante o discriminado & 157 & 20,7 & 315 & 41,6 \\
Abuso Físico & & & & \\
Ha sufrido amenazas de agresión física & 14 & 1,8 & 9 & 1,2 \\
Ha temido por su integridad física & 16 & 2,1 & 11 & 1,5 \\
Intencionalmente ha sido expuesto a riesgos médicos & 18 & 2,4 & 10 & 1,3 \\
Le han arrojado objetos con intención de dañarlo & 4 & 0,5 & 4 & 0,1 \\
Le han empujado & 34 & 4,5 & 38 & 5,0 \\
Le han tirado el pelo & 2 & 0,3 & 4 & 0,5 \\
Le han golpeado & 10 & 1,3 & 12 & 1,6 \\
Le han pateado & 5 & 0,7 & 7 & 0,9 \\
Abuso sexual & & & & \\
Ha sufrido proposiciones sexuales indeseadas & 18 & 2,4 & 15 & 2,0 \\
Le han dado nombres de connotación sexual & 24 & 3,2 & 25 & 3,3 \\
Le han acariciado en forma que le incomoda & 33 & 4,4 & 26 & 3,4 \\
Amenazado con ser perjudicado académicamente si no & & & & \\
accede a actividad sexual & 1 & 0,1 & 0 & 0,0 \\
Le han forzado a presenciar actividad sexual & 5 & 0,7 & 0 & 0,0 \\
\hline
\end{tabular}

${ }^{1}$ Percepción de haber experimentado la situación abusiva planteada en una sola oportunidad. 2Percepción de haber experimentado la situación abusiva planteada en más de una oportunidad.

Tabla 2. Percepción de conductas abusivas según curso del estudiante (\%)

\begin{tabular}{|llcccc|}
\hline Curso & $\begin{array}{c}\text { Abuso } \\
\text { verbal* }\end{array}$ & $\begin{array}{c}\text { Abuso } \\
\text { psicológico* }\end{array}$ & $\begin{array}{c}\text { Tipo de abuso } \\
\text { Abuso } \\
\text { físico }\end{array}$ & $\begin{array}{c}\text { Abuso } \\
\text { sexual* }\end{array}$ & $\begin{array}{c}\text { Abuso } \\
\text { total* }\end{array}$ \\
\hline $2^{\circ}$ & 67,7 & 73,7 & 21,2 & 8,1 & 83,8 \\
$3^{\circ}$ & 79,8 & 77,7 & 16,3 & 11,0 & 88,3 \\
$5^{\circ}$ & 88,7 & 85,7 & 17,3 & 13,9 & 94,4 \\
$7^{\circ}$ & 89,0 & 86,9 & 18,6 & 32,4 & 95,2 \\
\hline
\end{tabular}

$* p<0,05$. 
ción a abuso verbal, psicológico y sexual; los pares en ser agresores físicos (Tabla 3).

Las mujeres percibieron más agresiones sexuales por parte de docentes de sexo masculino, y los hombres percibieron más agresiones sexuales de parte de personas de sexo femenino, tanto de docentes como de pares.

Efectos. El 78,1\% de los alumnos que percibieron abuso reportaron algún efecto, sin diferencias por género, siendo los más frecuentemente mencionados la salud mental y la imagen que tenían del médico. Al estratificar por género, aparecen significativamente más afectadas las mujeres en salud mental, salud física, vida social, vida familiar y en considerar abandonar la carrera (Tabla 4). Un número significativo de los estudiantes que han percibido sufrir una situación abusiva ha pensado en abandonar la carrera de medicina (32,2\%). El 8,9\% respondió que cree que el efecto será permanente.

Reporte espontáneo adicional a las viñetas de la encuesta. Al preguntar por otras formas de abuso, los estudiantes mencionaron:

* humillar a los estudiantes que han repetido curso,

* humillar y descalificar frente a pacientes o estudiantes,

Tabla 3. Porcentaje de percepción de conductas abusivas según agresor

\begin{tabular}{|c|c|c|c|c|}
\hline & Docentes & $\begin{array}{c}\text { Otros } \\
\text { profesionales }\end{array}$ & $\begin{array}{c}\text { Personal } \\
\text { auxiliar }\end{array}$ & Alumnos \\
\hline Abuso verbal* & 73,6 & 15,7 & 7,7 & 20,9 \\
\hline Le han gritado & 32,2 & 6,2 & 4,1 & 8,2 \\
\hline Llamado en forma abusiva & 13,7 & 2,5 & 0,8 & 9,6 \\
\hline Dicho garabatos en forma ofensiva & 3,7 & 0,7 & 0,4 & 6,1 \\
\hline Humillado & 66,4 & 12,0 & 5,2 & 8,3 \\
\hline Abuso psicológico* & 71,2 & 18,0 & 6,7 & 32,5 \\
\hline Perjuicio académico & 26,6 & 0,5 & 0,3 & 0,0 \\
\hline Hablado mal de usted & 11,4 & 2,5 & 0,8 & 10,2 \\
\hline Asignado tareas injustas & 9,6 & 2,0 & 0,8 & 1,2 \\
\hline Acreditado con su trabajo & 11,4 & 4,8 & 0,3 & 16,2 \\
\hline Descalificado su trabajo & 37,8 & 3,7 & 0,9 & 9,1 \\
\hline Humillado o discriminado & 50,1 & 11,6 & 4,9 & 7,4 \\
\hline Abuso físico $§$ & 6,2 & 2,5 & 1,5 & 9,5 \\
\hline Amenazas de agresión física & 0,8 & 0,1 & 0,1 & 2,0 \\
\hline Temido por su integridad física & 0,7 & 0,3 & 0,3 & 2,2 \\
\hline Expuesto a riesgos médicos & 2,5 & 1,3 & 0,7 & 0,4 \\
\hline Arrojado objetos & 0,1 & 0,1 & 0,0 & 0,9 \\
\hline Le han empujado & 1,8 & 0,9 & 0,5 & 6,2 \\
\hline Le han tirado el pelo & 0,3 & 0,0 & 0,0 & 0,5 \\
\hline Le han golpeado & 0,7 & 0,3 & 0,0 & 2,0 \\
\hline Le han pateado & 0,1 & 0,0 & 0,0 & 1,5 \\
\hline Abuso sexual* & 8,9 & 1,6 & 1,3 & 5,9 \\
\hline Proposiciones sexuales indeseadas & 2,5 & 0,3 & 0,4 & 1,6 \\
\hline Nombres de connotación sexual & 3,0 & 0,5 & 0,5 & 2,2 \\
\hline Acariciado & 5,0 & 1,1 & 0,5 & 1,8 \\
\hline Perjuicio académico & 0,1 & 0,1 & 0,0 & 0,0 \\
\hline Presenciar actividad sexual & 0,3 & 0,0 & 0,0 & 0,7 \\
\hline
\end{tabular}

*Mayor frecuencia de parte de docentes, seguido por alumnos, $p<0,05$.

$\S$ Mayor frecuencia de parte de alumnos, seguido por docentes, $p<0,05$. 
Tabla 4. Efectos de las situaciones abusivas según género

\begin{tabular}{|lrrrrrr|}
\hline & \multicolumn{2}{c}{ Mujeres } & \multicolumn{2}{c}{ Hombres } & Total \\
& $\mathrm{n}$ & $\%$ & $\mathrm{n}$ & $\%$ & $\mathrm{n}$ & $\%$ \\
\hline Salud física & $38^{*}$ & 14,8 & $18^{*}$ & 7,3 & 56 & 11,1 \\
Salud mental & $217^{*}$ & 69,8 & $152^{*}$ & 53,5 & 369 & 62,0 \\
Vida social & $115^{*}$ & 42,8 & $86^{*}$ & 32,6 & 201 & 37,7 \\
Vida familiar & $79 *$ & 29,8 & $46^{*}$ & 18,1 & 125 & 24,1 \\
Calidad del trabajo & 156 & 55,1 & 130 & 48,9 & 286 & 52,1 \\
Imagen del médico & 186 & 63,7 & 161 & 59,9 & 347 & 61,9 \\
Abandonar la carrera & $102^{*}$ & 36,4 & $71^{*}$ & 27,5 & 173 & 32,2 \\
Algún efecto & 284 & 79,8 & 250 & 76,2 & 534 & 78,1 \\
\hline
\end{tabular}

*p $<0,05$.

* no respetar horarios, ni considerar tiempos de traslado entre centros asistenciales,

* catalogar mal a todo el curso por la falta de unos pocos,

* abusar económicamente al exigir pago de comidas en turno.

Se mencionan situaciones que podrían catalogarse como «acoso sexual»hacia alumnas: invitaciones a salir por parte de un docente, bromas poco delicadas, incomodidad por la forma de ser mirada 0 preguntar a las estudiantes por sus hábitos sexuales.

Algunas estudiantes se quejan de preparar un pastel para el turno», o de «avar la loza por ser la única mujer», o recibir comentarios tales como «a mujer inteligente es la que se queda callada»o que «debieran estudiar peluquería o diseño en vez de medicina».

Entre los otros efectos señalados aparece el sufrimiento vicario por presenciar situaciones abusivas hacia pacientes o compañeros y perder la capacidad de distinguir los límites de lo correcto».

\section{DisCUSIÓN}

Los resultados de este estudio, en que $90,9 \%$ de los estudiantes de medicina de la Universidad de Chile reportaron haber sufrido al menos un incidente abusivo durante la carrera, concuerdan con lo reportado en el estudio piloto, así como en otros estudios realizados en el extranjero y en Chile ${ }^{1,2,7}$.
La encuesta mide percepciones de los estudiantes y no hechos objetivables. Las percepciones son relevantes porque impactan la manera de ver y actuar del individuo en el mundo ${ }^{19}$. Al igual que en el estudio de Sheehan, no se realizó intento alguno de objetivar las situaciones percibidas como abusivas, ya que éstas ocurren en la intimidad de una relación ${ }^{7}$. Además, lo que un estudiante percibe como abusivo puede no serlo en otro contexto, o en un sentido ético o legal ${ }^{1,9}$.

Se podría suponer que la situación abusiva fue de suficiente relevancia como para recordarla y reportarla en la única medición realizada. El tiempo transcurrido puede distorsionar en más o en menos el reporte en una encuesta, introduciendo sesgos de memoria. Para controlar este error sistemático, se usaron categorías de respuestas amplias, sin detallar el número de veces del evento. Se consideró que el recuerdo de un único hecho abusivo podía ser algo aislado o anecdótico, pero su reporte «más de una vez», lo convertía en un hecho repetido y por ello de mayor trascendencia.

En cursos sucesivos, la percepción de conductas abusivas va en aumento. Se podría suponer que existe un efecto sumatorio en que los estudiantes de cursos superiores están expuestos durante más tiempo a sufrir situaciones de abuso. El abuso físico no comparte esta tendencia y es el único tipo de abuso en que los agresores principales son los alumnos. Se podría plantear que el abuso físico proveniente de pares es mayor en los cursos inferiores debido a la tradición del «mecho- 
neo» (ritual de iniciación al ingreso a la universidad).

En séptimo año se evidencia un incremento notorio en la percepción de abuso sexual, lo que se podría explicar por el aumento del contacto individual docente-estudiante y las largas horas de trabajo propias del internado.

Los alumnos reportan que el percibir un trato abusivo los afecta en aspectos importantes de su vida. En lo que se refiere al ámbito profesional, los afecta en la imagen que tenían del médico y $32,2 \%$ ha llegado a pensar en abandonar la carrera. No hay evidencias que estos estudiantes hayan concretado este pensamiento. Estos resultados concuerdan con estudios realizados en Chile y en el extranjero, donde se menciona que las situaciones abusivas van minando el entusiasmo y energía del estudiante, tornándolo en un ser apático y cínico ${ }^{1,2,7}$ (e Ibáñez P.). El 8,9\% responde que los efectos pueden ser permanentes, lo que nos parece una medida indirecta de la gravedad percibida de la situación abusiva.

No se conoce lo que ocurre en otras profesiones. Aun así, parece importante resaltar que estas situaciones, en el contexto de la educación médica, pueden tener un grave impacto. Los estudiantes durante la carrera de medicina adquieren tanto conocimientos como actitudes, habilidades y des-

\section{REFERENCIAS}

1. Silver HK, Guicken AD. Medical student abuse. Incidence, severity and significance. JAMA 1990; 263: 527-32.

2. Maida AM, Vásquez A, Herskovic V, Calderón JL, Jacard M, Pereira A et al. A report on student abuse during medical training. Med Teach 2003; 25: 497-501.

3. PetтाT B. Medical Student concems and fears before their third-year surgical clerkhip. Am J Surg 2005; 189: 492-6.

4. Stecker T. Well-being in an academic environment. Med Educ 2004; 38: 465-78.

5. SIIVER HK. Medical students and medical school. JAMA 1982; 247: 309-10.

6. Rosenberg D, Silver H. Medical student abuse. An unnecessary and preventable cause of stress. JAMA 1984; 251: 739-42. trezas y aprenden de sus profesores la forma en que se supone que los médicos se comportan»?0. Sin embargo, los docentes no siempre están conscientes de este poderoso rol de modelos ${ }^{20,21}$. Un profesor con actitudes abusivas ejerce una poderosa influencia sobre los estándares de conducta y práctica de quien está aprendiendo.

Esta es una de las razones por la cual la educación médica en el mundo está experimentando cambios, con la idea central de recuperar la imagen del médico como un profesional compasivo. La mayoría de las escuelas de medicina ha modificado o están en proceso de modificación de sus currícula, incluyendo temas humanistas, alentando la autonomía del estudiante y un cambio de rol en el docente ${ }^{17,21-23}$.

Sheehan dice que si queremos atraer a personas bien adaptadas y talentosas a la profesión médica, no podemos darnos el lujo de tener un ambiente que es percibido por algunos como hostil o abusivo». Es imperativo crear conciencia tanto en docentes como en estudiantes que las situaciones abusivas no tienen lugar en una escuela de medicina, ya que éstas tienen consecuencias en las personas que las sufren. Se debe realizar un esfuerzo conjunto para que estudiar medicina sea una actividad que enaltece y hace mejores a las personas.

7. Sheehan $\mathrm{KH}$, Sheehan DV, White $\mathrm{K}$, Leibowitz A, BALDwin DWC. A pilot study of medical student's abuse. Student perceptions of mistreatment and misconduct in medical school. JAMA 1990; 263: 533-7.

8. SCHUCHERT MK. The relationship between verbal abuse of medical students and their confidence in their clinical abilities. Acad Med 1998; 73: 907-9.

9. Richman JA, Flaherty JA, Rospena KM, Christensen ML. Mental health consequences and correlates of reported medical student abuse. JAMA 1992; 267: 692-4.

10. Carr P, Ash A, Friedman R, Szalacha L, Barnett R, Palepu A ET al. Faculty perceptions of gender discrimination and sexual harassment in academic medicine. Ann Intern Med 2000; 132: 889-96.

11. Oancia T, Bohm C, Carry T, Cujec B, Johnson D. The influence of gender and specialty on reporting of abusive and discriminatory behaviour by 
medical students, residents and physician teachers. Med Educ 2000; 34: 250-6.

12. WhITE GE. Sexual harassment during medical training: the perceptions of medical students at a university medical school in Australia. Med Educ 2000; 34: 980-6.

13. KasSebaum DG, CutLer E. On the culture of student abuse in medical school. Acad Med 1998; 73: 1149-58.

14. INTEBBI I. Indicadores psicológicos: mirar, escuchar y darse cuenta. En: Intebbi I. Abuso sexual infantil en las mejores familias. Granica. Buenos Aires, Argentina 1998; 173-201.

15. Consi J. Una mirada abarcativa sobre el problema de la violencia familiar. En: Corsi J ed. Violencia familiar: Una mirada interdisciplinaria sobre un grave problema social. Capítulo 1. Paidós, Buenos Aires, Argentina 1995; 15-63.

16. Bentovim A. Family violence: explanatory models to describe violent and abusive families. En: Bentovim A. Trauma organized systems. Chapter 2. Karnac Books. London, UK. 1992; 20.

17. Rosselot J. Hacia el médico que nuestros países necesitan: énfasis en la comunicación y en la formación de los docentes. Rev Méd Chile 2003; 131: 331-7.

18. Benitez G, Quintero B, Torres B. Prevalencia de riesgo de trastornos psiquiátricos en estudiantes de pregrado de la Escuela de Medicina de la P. Universidad Católica de Chile. Rev Méd Chile 2001; 129: 173-8.

19. BURR V. An Introduction to Social Constructivism. Routledge. London, UK 1999; 4-8.

20. HaRden RM, CRosby J. The good teacher is more than a lecturer- the twelve roles of a teacher. Med Teach 2000; 22: 334-47.

21. General Medical Council. Tomorrow's Doctors. Recommendations on undergraduate medical education. London, 1993 General Medical Council. Disponible en: www.gmc-uk.org/med_ed/ tomdoc.htm

22. WeAR D, CASTELIN B. The development of professionalism: Curriculum matters. Acad Med 2000; 75: 602-11.

23. The Medical School Objectives Writing Group. Learning objectives for medical student education- guidelines for medical schools: Report I of the Medical School Objectives Project. Acad Med 1999; 74: 13-8. 